\title{
HS1,2 Ig Enhancer Alleles Association to AIDS Progression in a Pediatric Cohort Infected with a Monophyletic HIV-Strain
}

\author{
Carla Montesano, ${ }^{1}$ Vincenzo Giambra, ${ }^{1,2}$ Domenico Frezza, ${ }^{1}$ Paolo Palma, ${ }^{3}$ \\ Eliseo Serone, ${ }^{4}$ Guido Castelli Gattinara, ${ }^{5}$ Maurizio Mattei, ${ }^{1}$ Giorgio Mancino, ${ }^{6}$ \\ Vittorio Colizzi, ${ }^{1}$ and Massimo Amicosante ${ }^{7,8}$ \\ ${ }^{1}$ Department of Biology, University of Rome "Tor Vergata," 00133 Rome, Italy \\ ${ }^{2}$ Terry Fox Laboratory, British Columbia Cancer Agency, Vancouver, BC, Canada V5Z 1L3 \\ ${ }^{3}$ University Department of Pediatrics, DPUO, Unit of Immune and Infectious Diseases, Children's Hospital "Bambino Gesù," \\ 00165 Rome, Italy \\ ${ }^{4}$ Istituto Mario Negri Sud, 66030 Chieti, Italy \\ ${ }^{5}$ International Training Unit, Children's Hospital "Bambino Gesù," 00165 Rome, Italy \\ ${ }^{6}$ San Pietro Fatebenefratelli Hospital, 00189 Rome, Italy \\ ${ }^{7}$ Department of Biomedicine and Prevention, University of Rome "Tor Vergata," 00133 Rome, Italy \\ ${ }^{8}$ ProxAgen Ltd., 1505 Sofia, Bulgaria
}

Correspondence should be addressed to Carla Montesano; montesano@uniroma2.it

Received 28 February 2014; Revised 28 April 2014; Accepted 28 April 2014; Published 20 May 2014

Academic Editor: Esteban Martinez

Copyright (C) 2014 Carla Montesano et al. This is an open access article distributed under the Creative Commons Attribution License, which permits unrestricted use, distribution, and reproduction in any medium, provided the original work is properly cited.

\begin{abstract}
Alteration in the humoral immune response has been observed during HIV infection. The polymorphisms of enhancer HS1,2, member of the $3^{\prime}$ regulatory region of the Ig heavy chain cluster, may play a role in the variation of the humoral response leading to pathological conditions. To assess the role of the HS1,2 polymorphic variants in the progression of AIDS, the HS1,2-A allelic frequencies were investigated in a cohort of HIV infected pediatric subjects from a nosocomial outbreak with a monophyletic strain of HIV. From a total group of 418 HIV infected children in the outbreak cohort, 42 nonprogressors and 31 progressors without bias due to antiretroviral therapy were evaluated. HS1,2 allele ${ }^{*} 1$ has been associated with nonprogressors (allelic frequency: $51.19 \%$ versus $33.87 \%$ in progressors, OR 0.5 , and $P=0.0437$ ), while allele ${ }^{*} 2$ has been associated with progression (allelic frequency: $48.39 \%$ versus $30.95 \%$ in nonprogressors, OR 2.1 , and $P=0.0393$ ). Further, only subjects carrying allele ${ }^{*} 2$ in absence of allele ${ }^{*} 1$, either in homozygous condition for allele ${ }^{*} 2$ [nonprogressors $2 / 42(4.76 \%)$, Progressors $7 / 31(22.58 \%)$, OR 5.8, and $P=0.0315$ ] or in combination with other allelic variants [nonprogressors 7/42 (16.67\%), Progressors 13/31 (41.93\%), OR 3.61, and $P=0.0321$ ], have been associated with HIV progression to AIDS. In conclusion, while the HS1,2 allele * 1 has a protective effect on HIV progression when present, allele ${ }^{*} 2$ is associated with progression toward AIDS when allele ${ }^{*} 1$ is absent.
\end{abstract}

\section{Introduction}

In HIV infected subjects, the progression to AIDS has been associated with a number of host genetic factors [1] and virological variants [2], suggesting that the individual immune-response to HIV might be influenced at many levels of the virus-host interaction.
The principal targets of HIV are the CD4+ T-lymphocytes whose decline causes the impairment of the host immune defences [3]. However, alteration in the humoral immuneresponse mediated by B-cells, including hypergammaglobulinemia $[4,5]$, has been observed during HIV infection in children and adults $[6,7]$ particularly in the late stages of disease. 


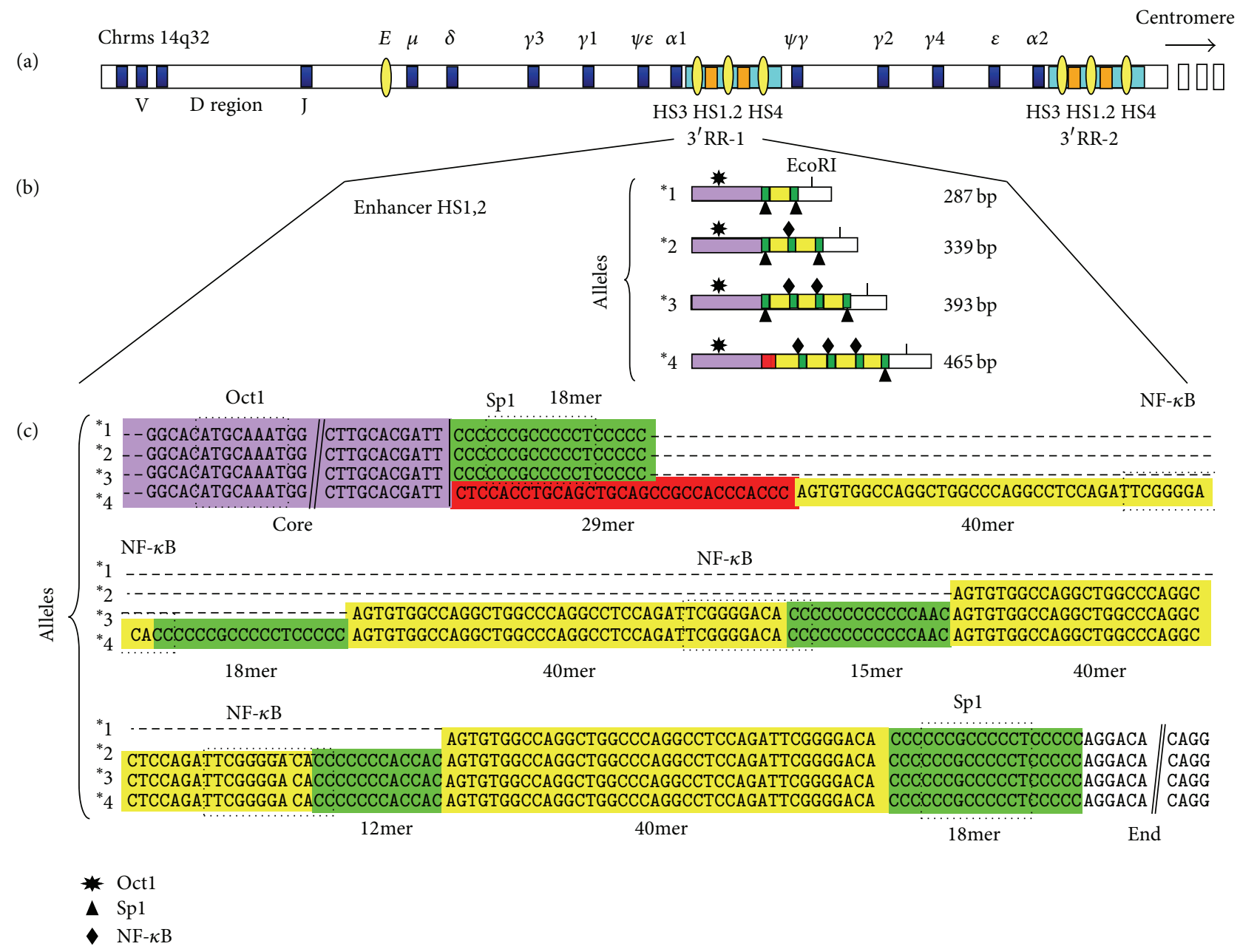

FIGURE 1: Schematic map of the constant heavy chain region: (a) location of the two regulatory regions within the heavy chain genes on chromosome 14q32; (b) enlargement of the $3^{\prime}$ RR-1 hs1.2 polymorphic enhancer and schematic representation of the four alleles; (c) nucleotidic sequence of the hs1.2 enhancer with the differences of the four alleles and the consensus for the Spl and NF-kB transcription factor consensus.

The humoral response is the result of interactions whose modulation is partially depending on polymorphic features of genes leading to Ig maturation and production. The $3^{\prime} \mathrm{Ig}$ heavy chain regulatory regions mainly control Ig heavy chain expression [8].

The $3^{\prime}$ regulatory region at the $3^{\prime}$ of the Ig $\alpha-1$ constant gene includes three enhancers among which only the central HS1,2-A is polymorphic (see Figure 1) [9]. This region is involved in B lymphocytes maturation and Ig production [10]. Furthermore, the different allelic variants have been associated with a different capability of controlling Ig levels [11].

The HS1,2-A alleles have a remarkable variability in frequency among world populations [12] and the allele ${ }^{*} 2$ variant of the HS1,2-A has been associated with susceptibility to several immune-related diseases [13-16], suggesting that it might contribute to pathological conditions.

A progressive impairment of $\mathrm{B}$ cell response to recall antigens, the increased polyclonal activation of B cells, and the expression of activation markers on their surface have been observed in HIV patients [4-7]. Because no specific B cell-related factors have been associated with HIV protection, in this study we investigated the frequency of the Ig $3^{\prime}$ enhancer HS1,2-A alleles in the progression toward AIDS. To minimize the influence of genetic background (due to a population originating from different or large geographic areas) and viral strain variability on HIV progression, we focused the analysis on a cohort of HIV infected subjects from a nosocomial outbreak with a monophyletic strain of HIV in the Benghazi Children Hospital in Libya [17].

\section{Patients and Methods}

2.1. Study Population. The cohort involved in the outbreak of HIV infection, at the "El-Fath Children's Hospital" of Benghazi, includes 418 children, 18 mothers, and 2 nurses. All the children were infected after hospitalization or after attending the hospital outpatients. The HIV infection, outbreak, immunological, and immunogenetics characterizations of the cohort have been previously described [17, 20-22], 
TABle 1: Demographic and clinical characteristics of the study groups classified according to clinical progression [18, 19].

\begin{tabular}{lccccc}
\hline & Patients & Sex (\% male) & $\begin{array}{c}\text { Median age at the first } \\
\left.\text { observation (IQR }{ }^{1}\right)\end{array}$ & $\begin{array}{c}\text { Median Nadir } \\
\text { \%CD4+ (IQR })\end{array}$ & $\begin{array}{c}\text { HIV viral load } \\
\text { median (range) }\end{array}$ \\
\hline Nonprogressors & 42 & $57.10 \%$ & $5.47(1.80-10.04)$ & $26.1 \%(24.0-31.0)$ & $650(50-17000)$ \\
Progressors & 31 & $58.06 \%$ & $3.88(2.18-6.55)$ & $15.1 \%(11-18)$ & $73000(5280-500000)$ \\
\hline
\end{tabular}

${ }^{1} \mathrm{IQR}:$ interquartile range.

TABLE 2: Allelic frequency of the HS1,2-A alleles in HIV-infected nonprogressor and progressor patients.

\begin{tabular}{|c|c|c|c|c|c|c|}
\hline \multirow{2}{*}{ Allele } & \multicolumn{2}{|c|}{ Nonprogressors (84 alleles) } & \multicolumn{2}{|c|}{ Progressors (62 alleles) } & \multirow{2}{*}{ O.R. } & \multirow{2}{*}{$P$ (Fisher's exact test) } \\
\hline & $N$ & Frequency & $N$ & Frequency & & \\
\hline 1 & 43 & 0.5119 & 21 & 0.3387 & 0.4884 & 0.0437 \\
\hline 2 & 26 & 0.3095 & 30 & 0.4839 & 2.0910 & 0.0393 \\
\hline 3 & 6 & 0.0714 & 4 & 0.0645 & 0.8966 & 1 \\
\hline 4 & 9 & 0.1071 & 7 & 0.1129 & 1.0610 & 1 \\
\hline
\end{tabular}

In bold significant $P$ values.

indicating in the cohort the presence of a defined cluster of HIV-1 clade A/G virus (CRF02_AG).

HS1,2-A typing was performed upon residual blood sample availability, after informed consent [21, 22], from a subgroup of 73 children. According to CDC classification and the literature $[18,19]$, patients were divided into nonprogressors $(N=42)$ and progressors $(N=31)$ toward AIDS. Table 1 summarizes the demographic and clinical characteristic of patients.

The cohort subgroup evaluated in this study does not differ substantially from the entire cohort for the mean value of $\mathrm{CD} 4$ cell counts, the viral load, and the classification groups of AIDS progression. Thus, as far as the infection is concerned, the patients can be considered a homogeneous pool of subjects.

2.2. DNA Extraction. DNA was extracted from the serum samples. Briefly, 300 microliters of serum was treated with proteinase $\mathrm{K}$ and phenol-chloroform by standard procedure. The supernatant was then reextracted with chloroform/isoamilic alcohol and processed by the "microcon 100" method (Millipore, Bedford MA, USA).

2.3. PCR Analysis and Genotyping. The PCR amplifications were performed with 50 nanograms of genomic DNA as described by Giambra et al. [23]. The two nested PCRs discriminate the two HS1,2-A and -B enhancers present in the regulatory regions at the $3^{\prime}$ of both constant $\alpha-1$ and $\alpha$ 2 genes and the different alleles were analysed by gel agarose $2.5 \%$ electrophoresis [23].

2.4. Statistical Analysis. Allelic, phenotypic, and genotypic frequencies data are expressed as percentages with odds ratio (OR) when appropriate. Comparisons between frequencies in the study groups have been performed by Fisher's exact test. Hardy-Weinberg equilibrium has been evaluated as previously described [12]. All the statistical analyses have been carried out with GraphPad Prism (GraphPad Software Inc., San Diego, CA) packages.

\section{Results and Discussion}

In HIV infection, host genetic factors [1, 21, 22, 24-27], mostly related to the impairment of $\mathrm{T}$ cell response or with the increase of an inflammatory status, have been associated with AIDS progression. Differently, no specific factors have been identified or associated with the progression of AIDS in terms of production of antibodies, although alteration in the humoral immune response, such as progressive impairment of response to recall antigens and hypergammaglobulinemia during late stage of HIV infection, has been observed [4-6], where Ig $3^{\prime}$ enhancer HS1,2-A might play a role.

The observed frequencies for the HS1,2 genotypes in the overall population are in agreement with Hardy-Weinberg equilibrium, $(P>0.9)$ suggesting that the population is homogeneous. Further, the HS1,2 allelic frequency distribution of this Libyan population coming from the area of Benghazi is not different from other Mediterranean populations (data not shown, [12]).

Table 2 shows the allelic frequency analysis in the study groups. A statistically significant increase of allele ${ }^{*} 1$ frequency has been observed in HIV nonprogressors (51.19\%, versus $33.87 \%$ in progressors, $P=0.0437$ ), while allele ${ }^{*} 2$ has been found to increase in progressors (48.39\%, versus $30.95 \%$ in nonprogressors, $P=0.0393$ ).

This observation is further supported by the genotypic analysis (Table 3 ), where a statistically significant increase of the $2 / 2$ genotype in HIV progressor subjects has been observed [nonprogressors $2 / 42(4.76 \%)$, progressors $7 / 31$ (22.58\%), $P=0.0315]$.

Finally, the frequency of subjects carrying allele ${ }^{*} 1$ resulted increased in nonprogressors ( $80.95 \%$ versus $58.06 \%$ in progressors, $P=0.0398$ ) (Table 4 ), whereas the frequency of subjects carrying allele ${ }^{*} 2$ did not differ between the two patient groups (Table 4), either for the small population size evaluated or for a potential protective effect of allele ${ }^{*} 1$. In fact, 
TABLE 3: Frequency of the HS1,2-A genotypes in HIV-infected nonprogressor and progressor patients.

\begin{tabular}{|c|c|c|c|c|c|c|}
\hline \multirow{2}{*}{ Genotypes } & \multicolumn{2}{|c|}{ Nonprogressors $N=42$} & \multicolumn{2}{|c|}{ Progressors $N=31$} & \multirow{2}{*}{ O.R. ${ }^{1}$} & \multirow{2}{*}{$P(\text { Fisher's exact test })^{1}$} \\
\hline & $N$ & Frequency & $N$ & Frequency & & \\
\hline $1 \backslash 1$ & 9 & 0.2143 & 3 & 0.0968 & 0.3929 & 0.2169 \\
\hline $1 \backslash 2$ & 17 & 0.4048 & 10 & 0.3226 & 0.7003 & 0.6244 \\
\hline $1 \backslash 3$ & 3 & 0.0714 & 2 & 0.0645 & 0.8966 & 1 \\
\hline $1 \backslash 4$ & 5 & 0.1190 & 3 & 0.0968 & 0.7929 & 1 \\
\hline $2 \backslash 2$ & 2 & 0.0476 & 7 & 0.2258 & 5.8333 & 0.0315 \\
\hline $2 \backslash 3$ & 2 & 0.0476 & 2 & 0.0645 & 1.3793 & 1 \\
\hline $2 \backslash 4$ & 3 & 0.0714 & 4 & 0.1290 & 1.9259 & 0.4484 \\
\hline $3 \backslash 3$ & 0 & 0 & 0 & 0 & NA & NA \\
\hline $3 \backslash 4$ & 1 & 0.0238 & 0 & 0 & NA & 1 \\
\hline $4 \backslash 4$ & 0 & 0 & 0 & 0 & NA & NA \\
\hline
\end{tabular}

${ }^{1}$ NA: not applicable.

In bold significant $P$-values.

TABLE 4: Frequency of subjects carrying at least one copy of the HS1,2-A alleles in HIV-infected nonprogressor and progressor patients.

\begin{tabular}{lcccccc}
\hline \multirow{2}{*}{ Allele } & \multicolumn{2}{c}{ Nonprogressors $N=42$} & \multicolumn{2}{c}{ Progressors $N=31$} & O.R. & $P$ (Fisher's exact test) \\
& $N$ & Frequency & $N$ & Frequency & 0.3258 & 0.0398 \\
1 & 34 & 0.8095 & 18 & 0.7406 & 2.1562 & 0.1477 \\
3 & 24 & 0.5714 & 23 & 0.1290 & 0.8889 & 1 \\
4 & 6 & 0.1429 & 7 & 0.2258 & 1.0694 & 1 \\
\hline
\end{tabular}

In bold significant $P$ values.

subjects carrying allele ${ }^{*} 2$, in the absence of allele ${ }^{*} 1$, resulted in significant increase in progressors $(13 / 31,41.93 \%)$ rather than nonprogressors $(7 / 42,16.67 \%$; OR $3.61, P=0.0321)$ (see also Table 3).

We have already observed in other immune pathologies a significant increase of the frequency of the enhancer HS1,2 allele ${ }^{*} 2[13,14]$, supporting the hypothesis that the specific allelic conformations might trigger the different immune response leading to the hypergammaglobulinemia or other critical responses to cell-cell interaction and signalling. This hypothesis was confirmed in selective $\operatorname{IgA}$ deficiency where HS1,2 polymorphism influences Ig serum production [15]. This is consistent with both in silico analysis and EMSA experiments demonstrating the presence of a consensus binding site for NF-kB in allele * 2 but not in allele * $1[11,25$, 28].

Consequently, the allele ${ }^{*} 2$, capable of recruiting NF$\mathrm{kB}$, might give a different efficiency to the $3^{\prime}$ regulatory region towards Ig transcription. Interestingly NF-kB through the consensus on promoters of Bruton tyrosine kinase (Btk) indirectly regulates also the $\mathrm{B}$ cells' activating factor (BAFF) and the B cells' survival in response to BCR activation [29]. Likewise higher level of plasma BAFF has been associated with progression status and hypergammaglobulinemia in HIV infected patients [30].

Moreover, the presence of NF-kB in allele ${ }^{*} 2$ might also be responsible of B cell activation by cytokines such as TNF $\alpha$, IL-6, IFN $\alpha$ produced in HIV viremic patients [6]. In turn the $B$ cells activated by inflammatory stimuli might themselves induce activation of other immune cells and contribute to HIV progression.

Alternatively, the presence (yet to be identified) of specific haplotypes, linked to allele ${ }^{*} 2$, might be at the basis of the progression to AIDS which is observed in subjects homozygous for allele ${ }^{*} 2$.

In conclusion, despite possible limitations related to the size of our population sample, this study sheds new light on the relevance of new genetic factors involved in B cell exhaustion, associated with progression of AIDS in children. The application of the model developed in this study to a larger population might contribute to further clarification of the mechanisms behind the association of genetic susceptibility of the HS1,2 with HIV-1 and to the definition of the role of this marker in the early identification of subjects with $\mathrm{HIV}$ infection more prone to progression to AIDS, ensuring a proper followup and treatment.

\section{Conflict of Interests}

The authors declare that there is no conflict of interests regarding the publication of this paper.

\section{Authors' Contribution}

Carla Montesano and Vincenzo Giambra contributed equally to the work described in the paper. 


\section{Acknowledgments}

This study has been supported in part by a project of the University of Rome "Tor Vergata" (ex 60\% MIUR, 2006) MA and MIUR PRIN no.20073RH73W003 DF. The authors would like to thank Mrs Angela Durland for editing the paper.

\section{References}

[1] M. Carrington, G. W. Nelson, M. P. Martin et al., "HLA and HIV-1: heterozygote advantage and $\mathrm{B}^{*} 35-\mathrm{Cw}^{*} 04$ disadvantage," Science, vol. 283, no. 5408, pp. 1748-1752, 1999.

[2] N. Casartelli, G. Di Matteo, C. Argentini et al., "Structural defects and variations in the HIV-1 nef gene from rapid, slow and non-progressor children," AIDS, vol. 17, no. 9, pp. 1291-1301, 2003.

[3] B. Rodriguez, A. K. Sethi, V. K. Cheruvu et al., "Predictive value of plasma HIV RNA level on rate of CD4 T-cell decline in untreated HIV infection," The Journal of the American Medical Association, vol. 296, no. 12, pp. 1498-1506, 2006.

[4] A. De Milito, "B lymphocyte dysfunctions in HIV infection," Current HIV Research, vol. 2, no. 1, pp. 11-21, 2004.

[5] A. M. Levine, "Monoclonal gammopathy associated with HIV infection," Clinical Infectious Diseases, vol. 43, no. 9, pp. 12061208, 2006.

[6] S. Moir and A. S. Fauci, "B cells in HIV infection and disease," Nature Reviews Immunology, vol. 9, no. 4, pp. 235-245, 2009.

[7] S. Pensieroso, A. Cagigi, P. Palma et al., "Timing of HAART defines the integrity of memory $\mathrm{B}$ cells and the longevity of humoral responses in HIV-1 vertically-infected children," Proceedings of the National Academy of Sciences of the United States of America, vol. 106, no. 19, pp. 7939-7944, 2009.

[8] E. Pinaud, A. A. Khamlichi, C. Le Morvan et al., "Localization of the $3^{\prime}$ IgH locus elements that effect long-distance regulation of class switch recombination," Immunity, vol. 15, no. 2, pp. 187199, 2001.

[9] E. Pinaud, M. Marquet, R. Fiancette et al., "The IgH locus $3^{\prime}$ regulatory region: pulling the strings from behind," Advances in Immunology, vol. 110, pp. 27-70, 2011.

[10] Y. Hu, Q. Pan, E. Pardali et al., "Regulation of germline promoters by the two human Ig heavy chain $3^{\prime}$ alpha enhancers," Journal of Immunology, vol. 164, no. 12, pp. 6380-6386, 2000.

[11] D. Frezza, V. Giambra, C. Mattioli et al., "Allelic frequencies of $3^{\prime}$ Ig heavy chain locus enhancer HS1,2-A associated with Ig levels in patients with schizophrenia," International Journal of Immunopathology and Pharmacology, vol. 22, no. 1, pp. 115-123, 2009.

[12] V. Giambra, C. Martínez-Labarga, M. Giufre' et al., "Immunoglobulin enhancer HS1,2 polymorphism: a new powerful anthropogenetic marker," Annals of Human Genetics, vol. 70, no. 6, pp. 946-950, 2006.

[13] D. Frezza, V. Giambra, R. Cianci et al., "Increased frequency of the immunoglobulin enhancer HS1,2 allele 2 in coeliac disease," Scandinavian Journal of Gastroenterology, vol. 39, no. 11, pp. 1083-1087, 2004.

[14] R. Cianci, V. Giambra, C. Mattioli et al., "Increased frequency of Ig heavy-chain HS1,2-A enhancer * 2 allele in dermatitis herpetiformis, plaque psoriasis, and psoriatic arthritis," Journal of Investigative Dermatology, vol. 128, no. 8, pp. 1920-1924, 2008.
[15] V. Giambra, R. Cianci, S. Lolli et al., "Allele * 1 of HS1.2 enhancer associates with selective IgA deficiency and IgM concentration," Journal of Immunology, vol. 183, no. 12, pp. 8280-8285, 2009.

[16] C. Aupetit, M. Drouet, E. Pinaud et al., "Alleles of the alpha1 immunoglobulin gene $3^{\prime}$ enhancer control evolution of IgA nephropathy toward renal failure," Kidney International, vol. 58, no. 3, pp. 966-971, 2000.

[17] T. de Oliveira, O. G. Pybus, A. Rambaut et al., "Molecular epidemiology: HIV-1 and HCV sequences from Libyan outbreak," Nature, vol. 444, no. 7121, pp. 836-837, 2006.

[18] Centers for Disease Control Prevention, "Revised classification system for human immunodeficiency virus infection in children less than 13 years of age," Morbidity and Mortality Weekly Report, vol. 43, pp. 1-19, 1994.

[19] A. Petrucci, M. Dorrucci, M. Alliegro et al., "How many HIV-infected individuals may be defined as long-term nonprogressors? A report from the Italian Seroconversion Study," Journal of Acquired Immune Deficiency Syndromes and Human Retrovirology, vol. 14, no. 3, pp. 243-248, 1997.

[20] S. Yerly, R. Quadri, F. Negro et al., "Nosocomial outbreak of multiple bloodborne viral infections," Journal of Infectious Diseases, vol. 184, no. 3, pp. 369-372, 2001.

[21] A. Grifoni, C. Montesano, P. Palma, A. Salerno, V. Colizzi, and M. Amicosante, "Role of HLA-B alfa-3 domain amino acid position 194 in HIV disease progression," Molecular Immunology, vol. 53, no. 4, pp. 410-413, 2013.

[22] C. Montesano, C. T. Bonanno, A. Grifoni et al., "Impact of human leukocyte antigen polymorphisms in human immunodeficiency virus progression in a paediatric cohort infected with a mono-phyletic human immunodeficiency virus-1 strain," Journal of AIDS and Clinical Research, vol. 5, article 282, pp. 2155-6113, 2014.

[23] V. Giambra, A. Fruscalzo, M. Giufre' et al., "Evolution of human IgH3'EC duplicated structures: both enhancers HS1,2 are polymorphic with variation of transcription factor's consensus sites," Gene, vol. 346, pp. 105-114, 2005.

[24] G. Kaur and N. Mehra, "Genetic determinants of HIV-1 infection and progression to AIDS: immune response genes," Tissue Antigens, vol. 74, no. 5, pp. 373-385, 2009.

[25] E. A. Trachtenberg and H. A. Erlich, "A review of the role of the human leukocyte antigen (HLA) system as a host immunogenetic factor influencing HIV transmission and course of infection with progression to AIDS," in HIV Molecular Immunology Database, B. T. Korber, C. Brander, B. F. Haynes et al., Eds., pp. 1-60, 2001.

[26] C. G. Anastassopoulou and L. G. Kostrikis, "The impact of human allelic variation on HIV-1 disease," Current HIV Research, vol. 1, no. 2, pp. 185-203, 2003.

[27] A. J. McMichael and S. L. Rowland-Jones, "Cellular immune responses to HIV," Nature, vol. 410, no. 6831, pp. 980-987, 2001.

[28] D. Frezza, B. Tolusso, V. Giambra et al., "Polymorphisms of the IgH enhancer HS1, 2 and risk of systemic lupus erythematosus," Annals of the Rheumatic Diseases, vol. 71, pp. 1309-1315, 2012.

[29] L. Yu, A. J. Mohamed, O. E. Simonson et al., "Proteasomedependent autoregulation of bruton tyrosine kinase (Btk) promoter via NF-kB," Blood, vol. 111, no. 9, pp. 4617-4626, 2008.

[30] B. Rodriguez, H. Valdez, W. Freimuth, T. Butler, R. Asaad, and M. M. Lederman, "Plasma levels of B-lymphocyte stimulator increase with HIV disease progression," AIDS, vol. 17, no. 13, pp. 1983-1985, 2003. 


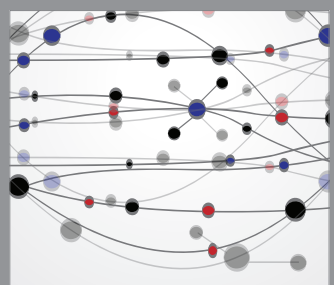

The Scientific World Journal
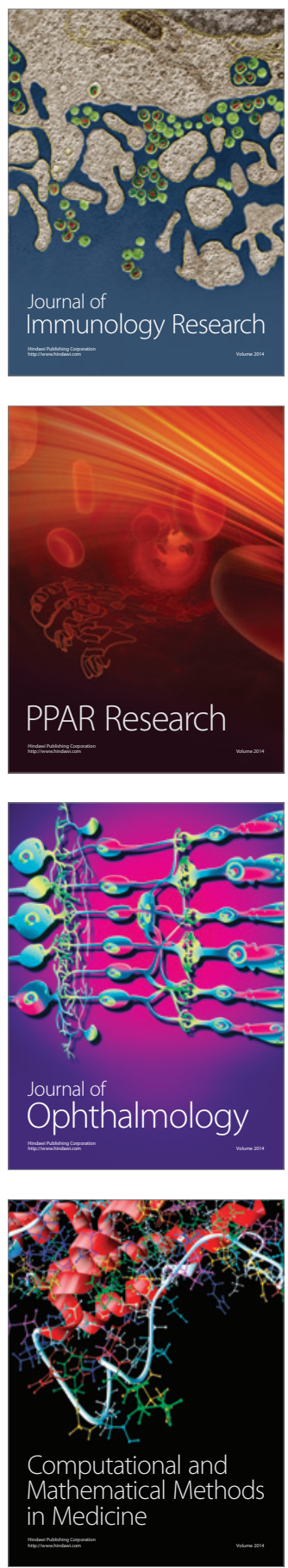

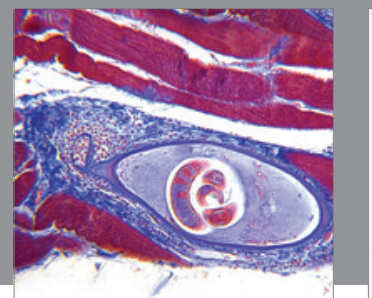

Gastroenterology

Research and Practice
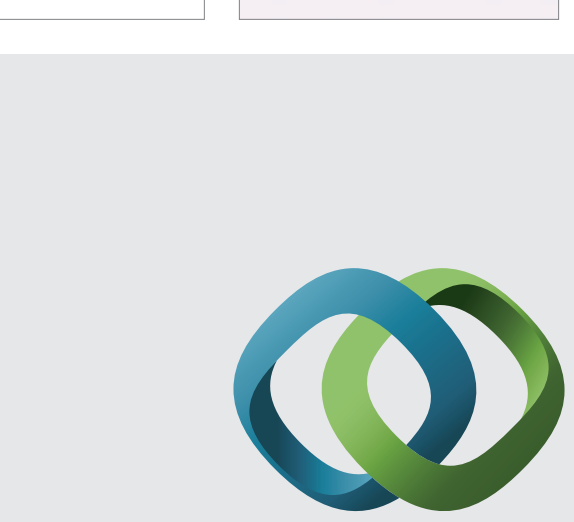

\section{Hindawi}

Submit your manuscripts at

http://www.hindawi.com
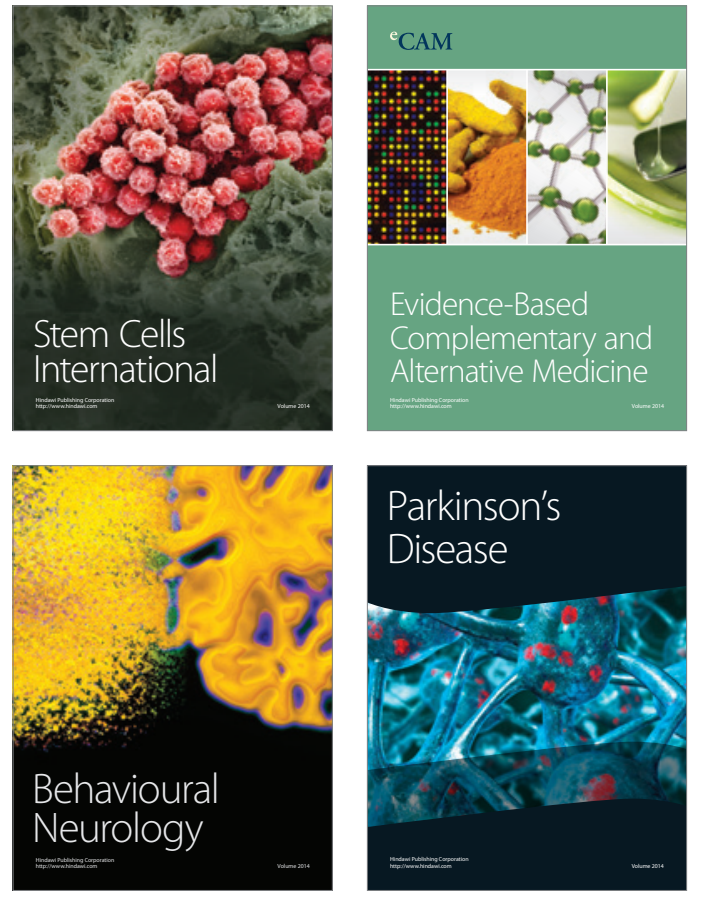
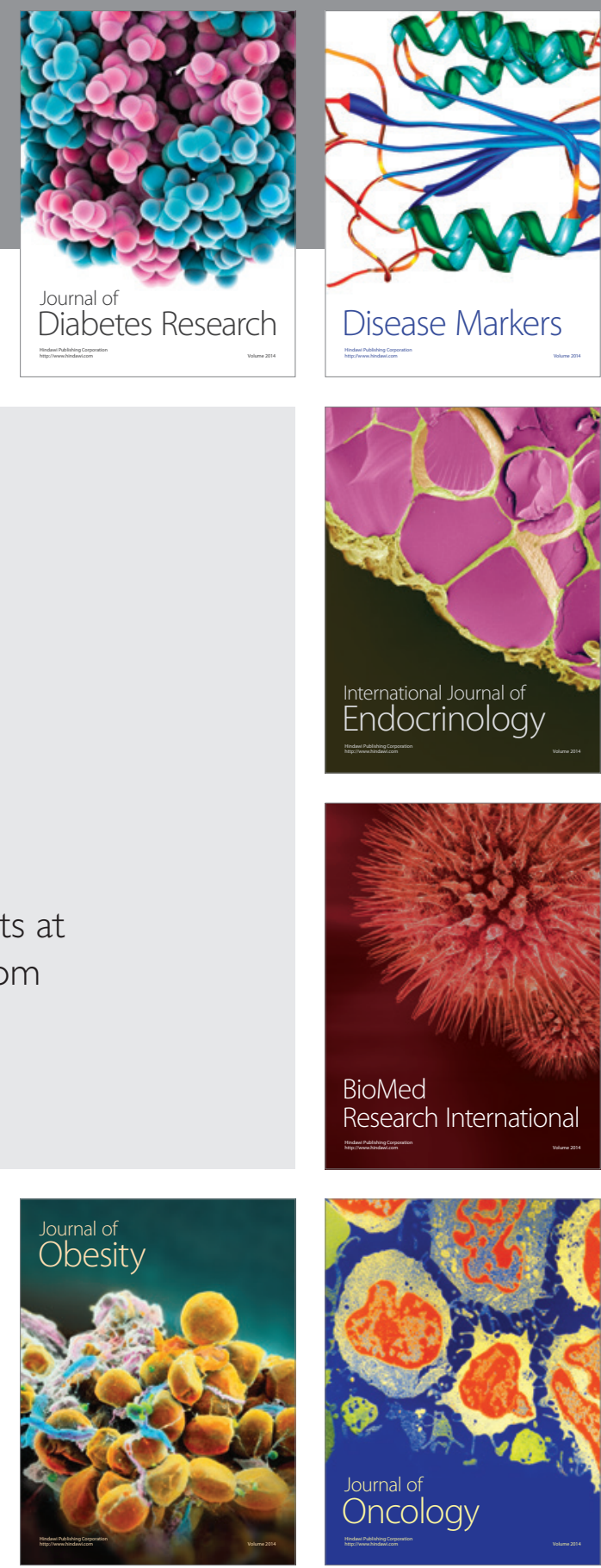

Disease Markers
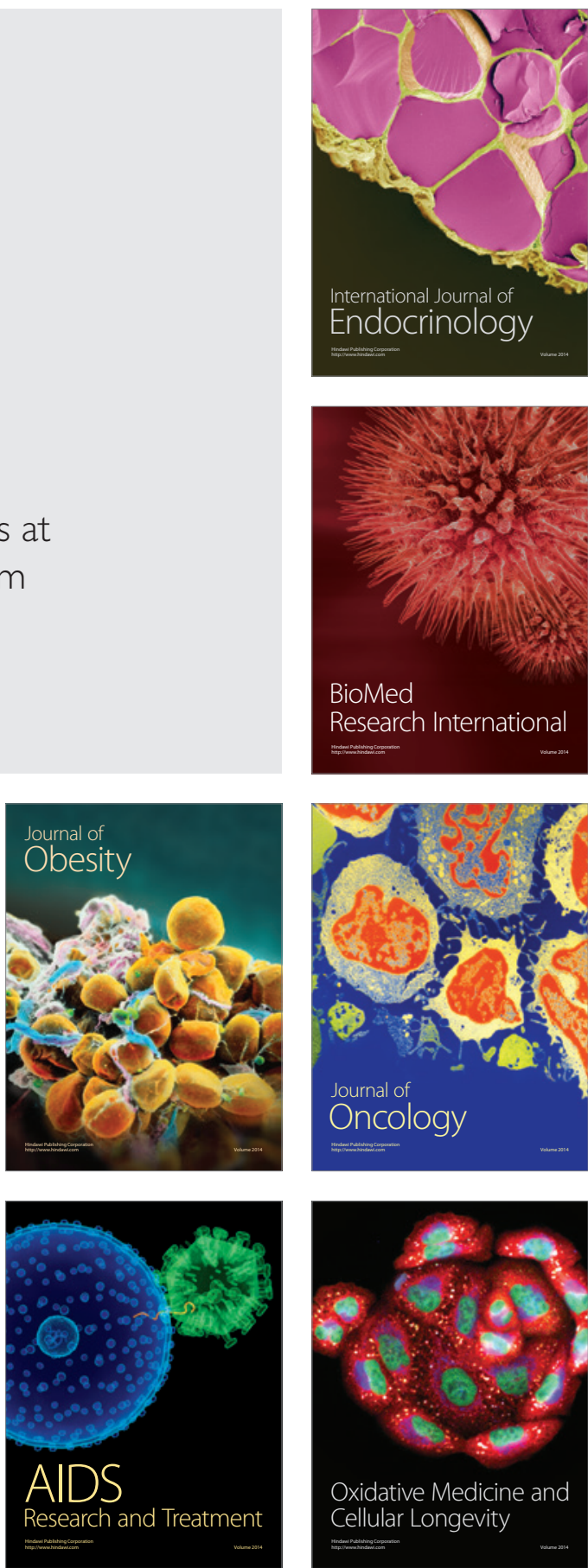